\title{
Differentiation between polaron-pair and triplet-exciton polaron spin-dependent mechanisms in organic light-emitting diodes by coherent spin beating
}

\author{
W. J. Baker, ${ }^{1}$ D. R. McCamey, ${ }^{1,2}$ K. J. van Schooten, ${ }^{1}$ J. M. Lupton, ${ }^{1,3,{ }^{*}}$ and C. Boehme ${ }^{1, *}$ \\ ${ }^{1}$ Department of Physics and Astronomy, University of Utah, Salt Lake City, Utah 84112, USA \\ ${ }^{2}$ School of Physics, University of Sydney, Sydney 2006, Australia \\ ${ }^{3}$ Institut für Experimentelle und Angewandte Physik, Universität Regensburg, Regensburg, Germany
}

(Received 22 August 2011; published 19 October 2011)

\begin{abstract}
Pulsed electrically detected magnetic resonance offers a unique avenue to distinguish between polaronpair (PP) and triplet-exciton polaron (TEP) spin-dependent recombination, which control the conductivity and magnetoresistivity of organic semiconductors. Which of these two fundamental processes dominates depends on carrier balance: by injecting surplus electrons we show that both processes simultaneously impact the device conductivity. The two mechanisms are distinguished by the presence of a half-field resonance, indicative of TEP interactions, and transient spin beating, the signature of PPs. Coherent spin Rabi flopping in the half-field (triplet) channel is observed, demonstrating that the triplet exciton has an ensemble phase coherence time of at least $60 \mathrm{~ns}$, offering insight into the effect of carrier correlations on spin dephasing.
\end{abstract}

DOI: 10.1103/PhysRevB.84.165205

PACS number(s): 71.20.Rv, 71.35.Gg

\section{INTRODUCTION}

Spin-dependent phenomena, though among the first physical effects studied in organic semiconductors, ${ }^{1}$ have only recently been explored in the context of device applications. ${ }^{2}$ Spin-dependent conductivity has received particular attention in magnetoresistive devices, ${ }^{2-7}$ which offer avenues to information storage and magnetic field sensing. However, without direct observation of spin precession through, e.g., the Hanle effect, it is hard to separate magnetic field phenomena in spin valves $^{2}$ into bulk spin polarization and surface magnetization effects. ${ }^{8}$ Devices with nonmagnetic electrodes also show magnetoresistance effects, ${ }^{3-7}$ which are virtually impossible to break down into bulk and interfacial processes. Although models of organic magnetoresistance have started appearing, largely based on site-specific Pauli-blocking mechanisms, ${ }^{5}$ the qualitative similarity in magnetoresistance in a wide range of very different semiconducting materials, ${ }^{2,9}$ both organic and inorganic, urges caution in assigning a particular material-specific mechanism to the phenomenon. Many of these models derive from a phenomenological description of the influence of magnetic fields on molecular reaction kinetics. ${ }^{10}$ As these approaches rely on indirect inference of the role of spin in conductivity, it is not always apparent how they may apply to a particular measurement situation. ${ }^{6}$ Electron paramagnetic resonance, in contrast, allows carrier spin to be directly manipulated and is thus ideally suited to unraveling spin-dependent transport in organic devices.

A longstanding question in the physics of spin-dependent processes in organic semiconductors has been the interpretation of optically or electrically detected magnetic resonance (O/EDMR) in terms of the polaron-pair (PP) mechanism or the exciton-polaron interaction. M. Wohlgenannt et al. have promoted the former, offering evidence for magnetic resonance signals arising from spin-dependent recombination and dissociation of weakly coupled spin-1/2 carrier pairs. ${ }^{11,12}$ In contrast Shinar et al. have reported clear signatures of half-field resonances in EDMR, which can only be observed if spin-1 species such as triplet excitons can influence conductivity, by, for example, modifying recombination and dissociation rates. ${ }^{13}$ The discussion has been particularly active ${ }^{14}$ because the assignment of the spin-dependent mechanism relates to the ultimate efficiency achievable in organic light-emitting diodes (OLEDs). ${ }^{7}$ We have recently explored how coherently driven spin dynamics impact spin-dependent transport using pulsed EDMR, the results of which have been broadly in agreement with the PP model. ${ }^{15-17}$ In the following, however, we show under which conditions both PP and triplet-exciton polaron (TEP) mechanisms ${ }^{18,19}$ can occur at once, providing an answer to the question of which mechanism is responsible for EDMR signals ${ }^{14}$ and highlighting the power of spin-resonance techniques in illuminating magnetic-field effects in organic semiconductors. $^{2-8}$

\section{EXPERIMENT}

\section{A. Devices: balanced and imbalanced injection}

We studied a commonly used conjugated polymer, namely poly[2-methoxy -5 - (2'- ethyl - hexyloxy )-1,4-phenylenevinylene] (MEH-PPV), incorporated into organic light emitting diode (OLED) structures designed to operate within an EDMR spectrometer, as described previously. ${ }^{16} \mathrm{We}$ tuned the electron-hole carrier balance within the device by either fabricating the OLED directly on an indium tin oxide (ITO) anode, or inserting a hole injection layer of poly(3,4-ethylenedioxythiophene) (PEDOT). ${ }^{20-22}$ In the former case, with a calcium cathode, the device is hole limited ${ }^{23}$ whereas PEDOT leads to more balanced carrier injection [Fig. 1(a) and 1(b)].

In order to experimentally verify the different injection schemes for the devices with and without the PEDOT holeinjection layer, a series of current-voltage (IV) measurements were undertaken. Figure 1(a) shows IV curves from devices both with the PEDOT ("balanced," dashed line) and without the PEDOT ("electron rich," solid line) layer. The electron-rich devices show consistently higher resistance than the balanced device at all temperatures. This is an indication of less injection 


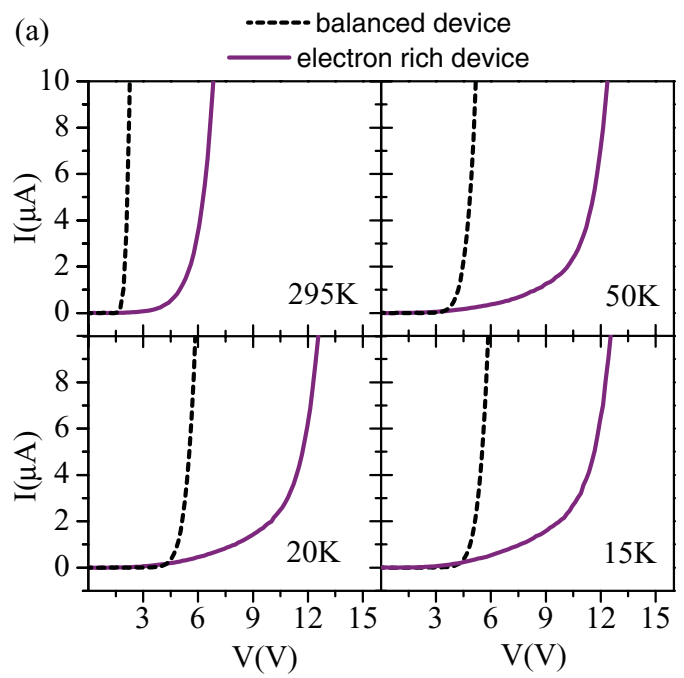

(b)

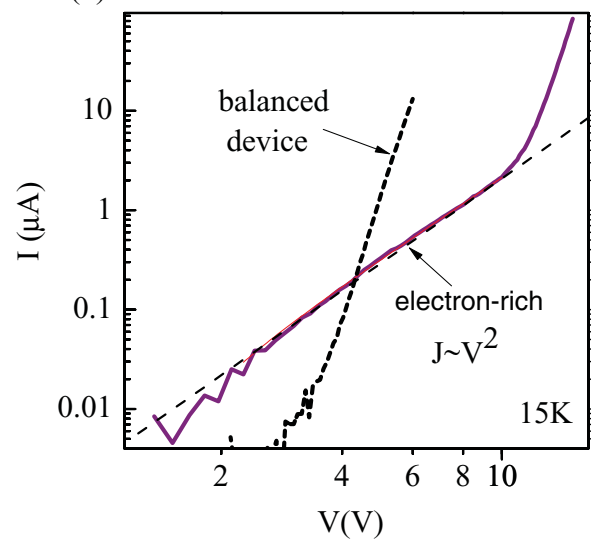

FIG. 1. (Color online) (a) IV curves of both balanced (dashed line) and electron-rich devices (solid line) showing a consistently higher current injection in the balanced devices over the imbalanced resulting from more effective hole injection as a result of the PEDOT. (b) The $15 \mathrm{~K}$ IV curve displays Child's law $\mathrm{V}^{2}$-dependent current from single carrier dominated injection of the devices without the PEDOT hole-injection layer.

into the devices without the PEDOT, as has been reported repeatedly in many previous OLED efficiency studies.

To further scrutinize the charge-injection imbalance, IV curves for both electron-rich and balanced devices measured at $15 \mathrm{~K}$ are shown on a double logarithmic scaling [see Fig. 1(b)]. The dramatic difference in the IV curve functionality between the two devices results from the difference in charge injection imbalance. The balanced device shows an exponential IV characteristic. The current in the electron-rich device shows a $\mathrm{V}^{2}$ dependency for voltages from 0-11 volts. This is expected in devices with space-charge limited current behavior, where the current is expected to follow the $\mathrm{V}^{2}$ dependence described by Child's law. The operating points for the EDMR measurement were always below $9 \mathrm{~V}$ in the experiment. The fact that these devices show space-charge limited current demonstrates the single carrier dominated transport. At higher applied voltages above $\sim 11 \mathrm{~V}$, the current dramatically increases, indicating the onset of significant hole injection. The functional behavior of the electron-rich IV balanced device

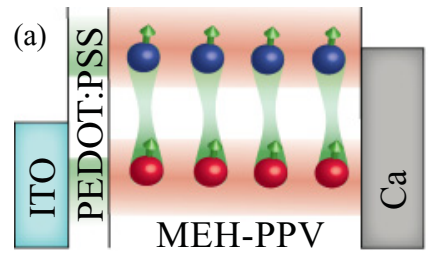

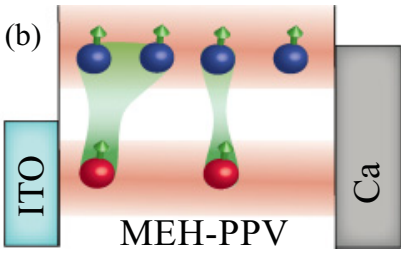

electron-rich device

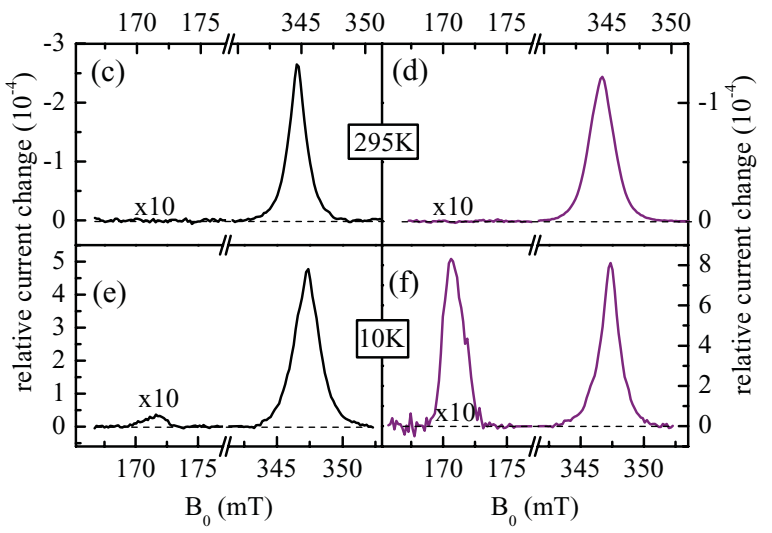

FIG. 2. (Color online) X-band EDMR of OLEDs with (a) carrier balance and (b) electron preference. (c), (d) At $295 \mathrm{~K}$, only the fullfield resonance is observed. (e), (f) At $10 \mathrm{~K}$, the half-field resonance is seen, which depends on carrier balance. Microwave frequencies are $9.66 \mathrm{GHz}$ at $295 \mathrm{~K}$ and $9.74 \mathrm{GHz}$ at $10 \mathrm{~K}$.

characteristic at high voltages approximately matches that of the balanced device at lower biases.

Figure 2 sketches both device configurations and presents $\mathrm{X}$-Band $(9.7 \mathrm{GHz})$ EDMR spectra at full and half-field at $295 \mathrm{~K}$ and $10 \mathrm{~K}$ for each device. The "relative change in current" is defined as $\Delta I / I_{0}$, where $\Delta I$ is the change in current on resonance due to spin-dependent processes and $I_{0}$ is the total current through the device when a constant bias is applied. All devices show full-field resonances in the differential current at approximately $345 \mathrm{mT}$, depending on the microwave frequency. At room temperature, no half-field resonance is observable (with a sensitivity of $\Delta I / I_{0}<10^{-7}$ ). Upon cooling to $10 \mathrm{~K}$, a resonance appears at half field $(\sim 172 \mathrm{mT}$, slightly lower than half of the full-field resonance due to the specific zero-field parameters, ${ }^{24}$ indicating the involvement of a spin-1 species in transport or recombination. The signal exhibits similar zero-field splitting parameters $(\mathrm{D} \approx 508 \mathrm{G}$ and $0 \leqslant \mathrm{E} \leqslant \mathrm{D} / 3$ ) to those observed by Shinar. ${ }^{13}$ This half-field signal is $10 \times$ stronger for the electron-rich device in Fig. 2(f), suggesting that the polaron partner of the triplet exciton in the TEP process is the electron. The spin- $1 / 2$ species can be clearly identified by their Rabi precession frequency in the EDMR transients, as subsequently discussed below.

\section{B. Multiple spin-dependent channels}

The main focus of this study is the identification and experimental discrimination of multiple spin-dependent channels in the electron-rich MEH-PPV OLED devices. Figure 3(b) 
balanced device electron-rich device

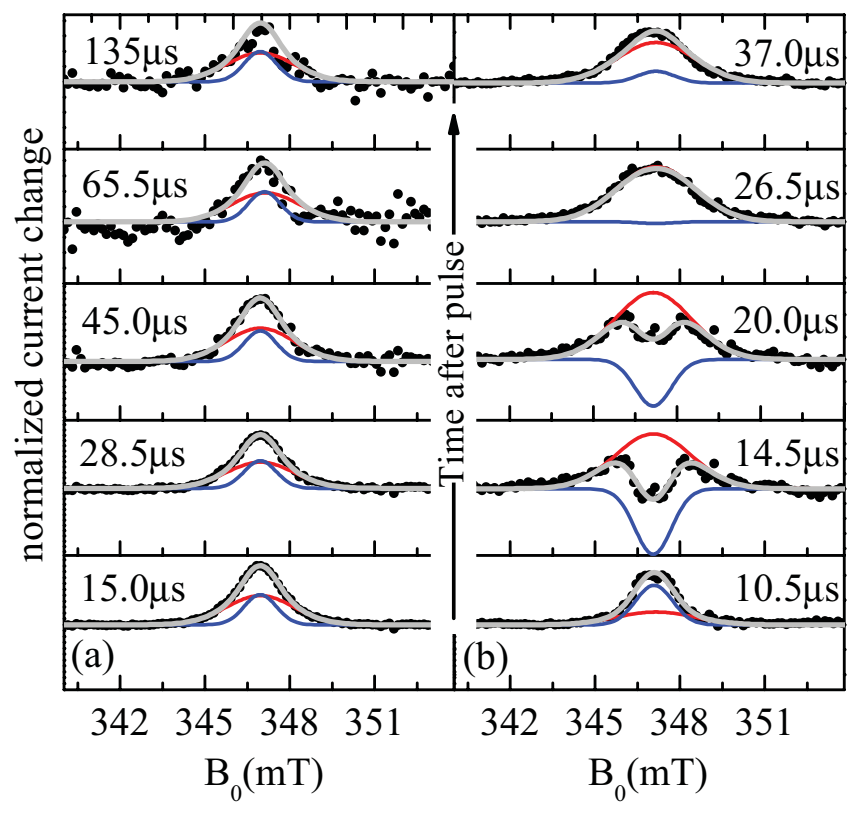

FIG. 3. (Color online) Transient EDMR spectra at different times after spin-resonant excitation at $10 \mathrm{~K}$. (a) In the balanced device the spectrum is time invariant and described by two Gaussians (see text). (b) With excess electrons the spectrum (described by the same two Gaussians) changes with time, indicating the presence of two spin-dependent mechanisms.

shows evidence that more than one spin-dependent channel exists due to the spectral line shape variation as a function of time after the spin-resonant microwave pulse. Regardless of injection imbalance, the resonances seen include both electrons and holes that have been inhomogeneously broadened by the hyperfine interactions with their random nuclear spin environments, giving a different Gaussian line width for each carrier type. ${ }^{16}$ If the majority of carriers are involved in the same spin-dependent process, such as in the PP signal seen in Fig. 3(a), the temporal dependence of the resonances involved in the spin-dependent process will be identical, and the compound line shape will not change with time. Consequently, the signal we see in Fig. 3(a) is a reflection of the overall pair dynamics and not just the singular dynamic information of any one of the pair partners. ${ }^{17}$ For instance if the longitudinal relaxation time, or $\mathrm{T}_{1}$, of one of the two pair partners changed, we might see an overall increase in dynamical behavior, but the temporal difference in the distributions would still be constant after the excitation, as it is determined by the dynamics of all the spins involved in the one spin-dependent channel.

We have previously discussed the transient response of an OLED current to a microwave pulse: the initial quenching and subsequent enhancement, due to the different recombination and dissociation dynamics of singlet and triplet PPs, is described in detail in Ref. 17. Figure 3(a) plots the resonance spectrum of the balanced device at different times following a microwave pulse $\left(B_{1} \approx 0.6 \mathrm{mT}\right)$ of $200 \mathrm{~ns}$ duration. For ease of comparison we plot normalized absolute values of the resonance: the lower four panels correspond to quenching, the upper panel to enhancement. The spectra can all be described by the sum of two Gaussians of different width, corresponding to electron and hole spin resonances broadened by the hyperfine interaction, as described in Ref. 16. The spectral shape does not change with time, demonstrating that only one spin-dependent mechanism dominates the resonance. For the electron-rich device in Fig. 3(b), however, the spectrum changes significantly with time but is still accurately described by the sum of the same two Gaussians whose relative amplitudes now vary with time. The current transient is more complex, showing the usual quenching-enhancement succession seen in balanced devices, as well as an additional enhancement at short times. For the electron-rich device, two spin-dependent mechanisms with different transient characteristics must be present to account for the temporal dynamics in the spectrum. Importantly, the amplitudes of the two mechanisms are of opposite sign: whereas one process gives rise to enhancement at, e.g., $14.5 \mu \mathrm{s}$, the other induces current quenching. It is important to understand that the transient data in Fig. 3(b) proves that the conductivity is influenced by two uncorrelated spin-dependent processes. However, it does not prove that either one of these channels is the TEP mechanism (see Appendix).

\section{Coherent "Rabi-beating" spectroscopy.}

To distinguish and understand the qualitative nature of the two spin-dependent mechanisms, we investigate the influence of the $\mathrm{B}_{1}$ microwave driving field duration and strength on the $\Delta I$ transient. The clearest evidence to date for the PP mechanism in spin-dependent processes has come from the observation of coherent spin-beating Rabi flopping in the current of a balanced OLED device. ${ }^{16}$ Since the PPs are weakly spin coupled, the application of an on-resonant microwave pulse $B_{1}$ drives the rotation of only a single spin- $1 / 2$ species within the pair if $B_{1}<\left|\Delta B_{\text {Hyp }}\right|$, the average difference in hyperfine field experienced by electron and hole. This rotation gives rise to a simple modulation of the current at the primary Rabi frequency $\gamma B_{1}$. If the magnitude of the driving microwave field $B_{1}$ exceeds $\left|\Delta B_{\mathrm{Hyp}}\right|$, both carriers precess in phase, and spin beating occurs at twice the Rabi frequency. It is this beating signature which offers a route to differentiating between the two mechanisms in the imbalanced devices.

The first demonstration of this beating effect was given by McCamey et al., ${ }^{16}$ who attributed this observation to PPs (weakly spin-coupled pairs of $s=1 / 2$ consisting of oppositely charged polaron states controlling recombination) in MEH-PPV; however, this effect has also been seen in currents of MEH-PPV/PCBM blends, ${ }^{25}$ where it was attributed to bipolaron (weakly spin-coupled pairs of $s=1 / 2$ consisting of equally charged polaron states controlling transport) in the MEH-PPV phase. The discrepancy between these two interpretations of the unambiguously observed pairs of $s=$ $1 / 2$ has recently been resolved by the test of both models using ODMR showing that the observed beat effect governs recombination and is therefore due to PPs with opposite charge. ${ }^{26}$ In the following we use the beat effect to distinguish spin-dependent recombination channels involving two carriers from those involving only one. Fig. 4(a) shows, for an electrondominated OLED, the enhancement-quenching-enhancement 


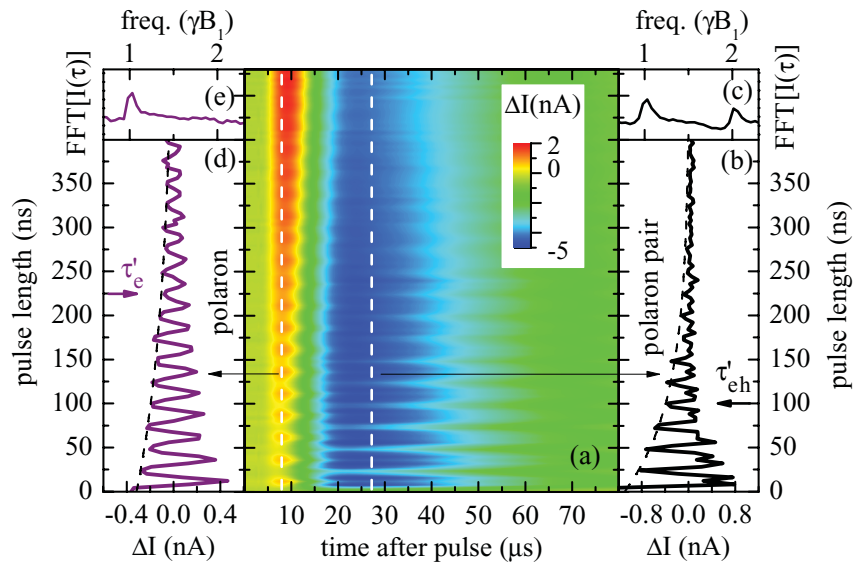

FIG. 4. (Color online) Rabi flopping in the current of an electrondominated device at $10 \mathrm{~K}$. The differential current on resonance $\left(\mathrm{B}_{0}=345.5 \mathrm{mT}\right)$ is plotted as a function of the microwave-pulse duration and time after the pulse. During the application of the microwave $\left(\mathrm{B}_{1}\right)$ field spin precession occurs, leading to Rabi flopping in the current along the vertical graph axis. At short times after $\mathrm{B}_{1}$ application single-frequency Rabi flopping is seen-the TEP mechanism (left panel; Fourier transform inset). At longer times, spin beating arises in the PP process (right panel) with a harmonic in the Fourier transform (top right).

$\Delta I(t)$ transient following the microwave pulse, as a function of the pulse length, for $B_{1}=1.4 \mathrm{mT}>\left|\Delta B_{\mathrm{Hyp}}\right|{ }^{16}$ Rabi flopping and beating is seen in the vertical slice of the plot at $27 \mu \mathrm{s}$ [Fig. 3(b)], consistent with the PP process. The lifetime of the Rabi oscillations is $\tau_{\text {eh }}^{\prime}=100 \mathrm{~ns}$, providing a lower limit for the spin-phase coherence time. The Fourier transform [Fig. 4(c)] shows two frequency components, which is characteristic of beating. In contrast, the vertical slice taken at $8 \mu \mathrm{s}$ [Fig. 4(d)] does not show beating: in this mechanism, which dominates at shorter times, only one spin-1/2 carrier is involved with a longer Rabi-flopping lifetime, $\tau_{\mathrm{e}}^{\prime}=225 \mathrm{~ns}$. The corresponding Fourier transform [Fig. 4(e)] reveals no higher harmonics in the spin precession. Spin beating, as for electron-hole pairs, is also conceivable for electron-electron or hole-hole pairs (bipolarons), although this would occur with different $\left|\Delta B_{\text {Hyp }}\right|$ magnitudes and can be excluded based on the data (see Appendix). We see no beating for driving fields exceeding $\left|\Delta B_{\text {Hyp }}\right|$, indicating that the signal is either due to a single spin-1/2 carrier, or occurs with a partner, which has a resonance at a substantially different magnetic field. The only other observed resonance in the system, the triplet half-field signal, is an obvious candidate. This hypothesis is supported by the time transient recorded following a microwave pulse resonant with the triplet at half field (not shown), which also leads to a current enhancement displaying a temporal dependence very similar to that of the additional enhancement seen at full field in the electron-rich device (Fig. 4). Note that the observations shown in Fig. 4, as well as the halffield resonance, safely exclude other spin-dependent transport processes that have been hypothesized in the literature (see Appendix). We find that only the properties of the TEP mechanism are consistent with the observed spin-dependent conductivity behavior.

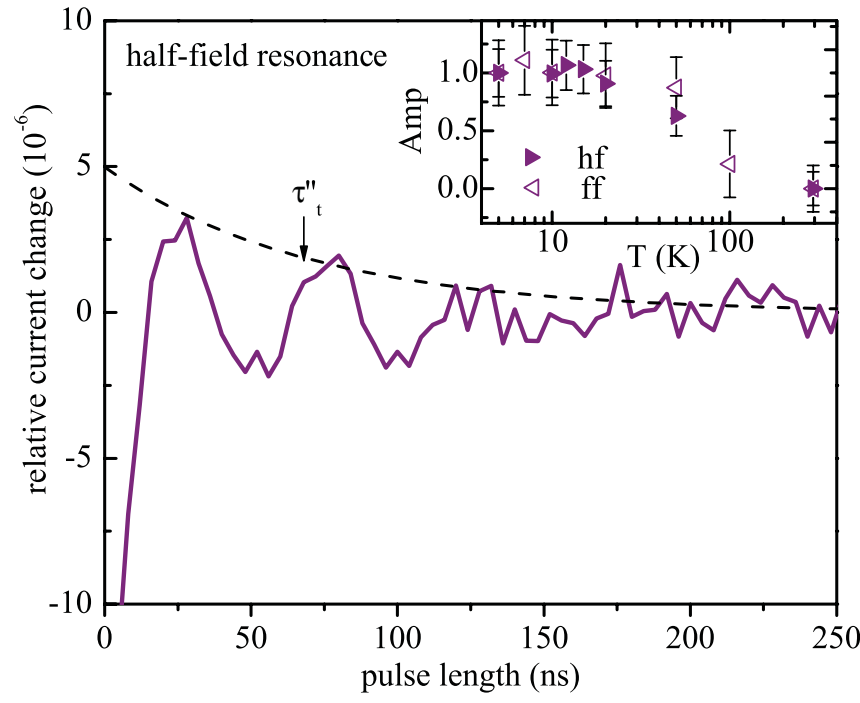

FIG. 5. (Color online) Spin-1 (triplet) half-field Rabi flopping in the electron-rich device. As the temperature increases, both the half-field resonance amplitude (hf) and the corrected amplitude of the nonbeating component of the full-field resonance (ff) disappear (upper inset).

\section{Half-field excitonic resonance}

In order to further test the weakly coupled TEP hypothesis, we can vary the temperature: at high temperatures, no half-field resonance is observed possibly because triplet excitons decay more readily by thermally activated nonradiative means, and the triplet density is therefore much lower. ${ }^{7}$ At full field, spin nutation is detected at both high and low temperatures; the $10 \mathrm{~K}$ nutation is shown in Fig. 4. At room temperature (not shown), where no half-field resonance is seen, the ratio between fundamental and harmonic amplitude in the full-field Rabi flopping depends solely on the strength of $B_{1}$ and the hyperfine interactions, ${ }^{16}$ which do not change with temperature. As the temperature is reduced, the part of the signal corresponding to spin-1/2 nutation increases and is clearly correlated with the increasing intensity of the half-field resonance (inset, Fig. 5), providing further evidence that the two signals arise from the same physical process, the TEP interaction. The points labeled (ff) in the Fig. 5 inset are amplitudes of the spin-1/2 full-field Rabi oscillation extracted from measurements of the Rabi flopping versus the real-time transient of the device current at each temperature (i.e., plots analogous to Fig. 4). These Rabi-oscillation measurements allow removal of the contribution from the PP process (which shows beating), resulting in a corrected spin-1/2 oscillation amplitude due exclusively to the single carrier (non-PP) process which does not show beating. With the TEP mechanism identified, we can now focus on the question of the actual spin phase coherence of the triplet exciton itself. Figure 5 shows half-field Rabi flopping in the OLED current as a function of pulse length. Here, only processes involving triplet excitons ( $\mathrm{S}=$ 1) are detected due to spin-resonant transitions between the triplet $\mathrm{T}_{+}$and $\mathrm{T}_{-}$levels. Both the full and half-field Rabi frequencies are proportional to $B_{1}$ (not shown), and the ratio of the frequencies $\Omega_{\text {Full }} / \Omega_{\text {Half }} \approx 2.1 \pm 0.7$. We are not aware 
of any prior discussion of the relation between full- and half-field Rabi frequencies in the literature. The coherence time extracted from the decay of the Rabi-oscillation amplitude $\tau^{\prime \prime}=60 \mathrm{~ns}$ is shorter than the single spin-1/2 time $\tau_{e}^{\prime}=225 \mathrm{~ns}$ noted in Fig. 4, indicating faster spin dephasing as expected in the strongly correlated electron-hole pair constituting the triplet exciton. The difference between these times contradicts the strongly spin-spin coupled triplet-PP (trion) hypothesis, ${ }^{14}$ which should exhibit identical dephasing times. However, the difference between $\tau^{\prime}$ and $\tau^{\prime \prime}$ is consistent with a weakly spinspin coupled pair comprising a triplet exciton and a polaron. As the zero-field matrix of the triplet exciton is strongly anisotropic, most of the randomly oriented triplet states will be slightly off-resonance under half-field excitation, and thus coherent dephasing of the triplet ensemble significantly faster than the dephasing of the polaron.

\section{CONCLUSIONS}

In conclusion we have identified a conductivity signature of the TEP process in MEH-PPV which becomes significant below $\sim 100 \mathrm{~K}$, when the triplet exciton lifetime becomes sufficiently long. Under magnetic resonance conductivity can be manipulated because of this process. Measuring the phase coherence and spin lifetimes of the triplet exciton using this approach offers a way to directly study the role and influence of the often elusive triplet excitons in purely hydrocarbon organic semiconductors. The direct coherent manipulation of the triplet exciton (Fig. 5) combined with electrical readout provides an avenue to utilize quantum properties of the spin degree of freedom in the limit of extremely strong exchange coupling, which is not available in inorganic semiconductors

\section{ACKNOWLEDGMENTS}

Acknowledgment is made to the Department of Energy Grant No. DESC0000909 for support of this research. J.M.L. would like to thank the David and Lucile Packard Foundation for financial support. D.R.M. acknowledges support through an Australian Research Council Postdoctoral Felloship (DP1093526).

\section{APPENDIX: EXCLUSION OF MODELS}

With the evidence of different spin-dependent channels given, coherent spin-Rabi nutation experiments are conducted in order to elucidate the nature of the signals (see Fig. 4 for data). One of the observed nutation signatures is in agreement with the PP mechanism, identical to the sole process seen in the charge-balanced device. The nutation signature of the other process is in agreement with the TEP process. The observed data refutes alternative explanations for this second observed nutation signature. As explained in above, the spin-nutation experiments produced the following observations. In the electron-rich device a strong "half-field" resonance is detected, which is indicative of a strongly coupled triplet exciton. At full field in the electron-rich device, a strong single spin-1/2 signal (Fig. 4) dominates the transient current behavior at $\sim 8 \mu$ s after a resonant microwave pulse. This is not seen in the balanced device. Reducing the temperature leads to an increase in the half-field resonance and a correlated increase in the full-field spin-1/2 signal (Fig. 5, inset). The inconsistency of these observations with spin-dependent mechanisms other than the triplet-polaron mechanism is discussed in the following.

\section{Single spin-1/2 process}

With the observation of the single spin-1/2 signal at full field, the first and simplest model to consider would be a spin-dependent process involving only the single spin-1/2 carrier. This may be due, for example, to spin resonantly manipulated hopping rates. Transport in disordered organic materials is governed by thermally assisted hopping through localized molecular sites. Due to the intrinsic disorder in the material, the next nearest hopping site will be energetically offset by some amount $\varepsilon$. If this offset energy is more than the thermal energy $\mathrm{kT}$ then that hop is energetically unfavorable. However, with the application of an external magnetic field this offset could be partially compensated by the Zeeman splitting, thus leading to a greater probability of a hopping transition with a rate $\Gamma$ that is inversely proportional to the energy offset between the hopping sites. The population of these Zeeman states will follow Boltzmann statistics, so the change in the conductivity due to driven transitions between the states will increase as the temperature decreases.

The signal for a spin-dependent process resulting from a single spin must be proportional to the square of the thermally driven spin polarization. ${ }^{27}$ This expected polarization dependence is not seen experimentally (Fig. 5, inset); instead, a constant value which persists over a large temperature range is seen. Furthermore, the signal strengths predicted by a single spin model are more than two orders of magnitude too small ${ }^{27}$ when compared to the signals seen in these experiments. Thus, a spin-dependent process involving a single spin cannot explain the data and, therefore, it can be excluded. This model does also not predict the presence of a signal at half field.

\section{Second PP process with different dynamics}

One hypothesis to account for the presence of a second spin-dependent channel next to the previously confirmed PP signal is to assume the presence of a second PP channel with different recombination dynamics. In the electron-rich devices a shift of the recombination zone toward the anode could also imply a shift of electronic and spin-relaxation times. Since PPs consist of two weakly coupled spins with $s=1 / 2$ similar to the bipolaron pairs, the same arguments exclude this possibility. The observed spin-dependent process should display beating at $\mathrm{B}_{1}$ fields above $1.1 \mathrm{mT}$ at the most. This is not seen experimentally, and thus this hypothesis can be excluded.

\section{Bipolaron}

The bipolaron interaction ${ }^{5}$ involves the weakly coupled pair of two identical carriers. The model consists of a free carrier passing through an already occupied site. The mobility through 
the site is proportional to the overall singlet content of the pair, leading to a Pauli-blocking mechanism. This interaction could become more prevalent in the case where there is a large carrier imbalance. We would expect different dynamics than for the PP mechanism, and thus a clearly distinguishable second signal would be seen. This model involves the interaction of two weakly coupled spins in pairs of either two electrons or two holes (pair of two $s=1 / 2$ ), and a beating in the Rabi-nutation signal should be seen once the driving field is comparable to the average difference in the local hyperfine fields between the pairs. Since the pair consists of either e-e or $h-h$, this average difference would be approximately the width of the respective resonance. In these experiments it is the narrower resonance that displays the additional Rabi nutation signal. This resonance has a width of $\sim 8 \mathrm{G}$, and after the application of a driving field of more than $14 \mathrm{G}$ the signal still shows no beating component. Thus, the bipolaron mechanism can be excluded as the origin of the observed signal. As with the single spin model, the triplet exciton resonance seen in the data is not predicted by the bipolaron model, either.

\section{Trion recombination}

The trion $^{28}$ or charged-exciton is usually described in terms of an exciton and "free" charge residing on the same molecular chain. This high degree of proximity between the two entities leads to a strong exchange coupling. Under spin resonance both a full-field and half-field resonant change in detected current would be observed, as the overall transition rate is proportional to the spin- $1 / 2$ or "doublet" content of the combined spin- $1+$ spin-1/2 (or spin-3/2) wavefunction. This rate can be manipulated by either driving the triplet or the polaron resonances of the trion, although due to the coupling, both the exciton and polaron will always nutate. The observed current change would be a result of an excited polaron with higher mobility after the Auger-like process. However, due to the strong exchange coupling, the Rabi nutation expected for this $s=3 / 2$ system at full field would not simply reflect the rotation of a single polaron with frequency $\gamma \mathrm{B}_{1}$. Instead, the nutation would occur with a primary frequency of $\sqrt{3} \gamma B_{1} \cdot{ }^{29}$ An $s=1 / 2$ nutation at $\gamma \mathrm{B}_{1}$ is not present in this system (see Fig. 4), and thus this hypothesis can be excluded.

\section{Triplet-triplet exciton annihilation}

At high triplet-exciton concentration, triplet-triplet interactions are expected ${ }^{1}$ and have indeed been reported before in organic devices. The interaction can change many experimental observables, including both the current and the luminescence. Under resonance of the triplet pair, there is a certain probability to annihilate the triplets and produce, due to spin conservation, a singlet ground state along with a weakly coupled PP. This would modify the current by changing the density of the overall PP population and thus would be observed through the intrinsic differences in triplet and singlet polaron dissociation rates. If the triplets were interacting as a weakly coupled pair, or the fine-structure term of the overall triplet-triplet Hamiltonian were negligible, the Rabi-nutation frequency expected at full field would be due to the transition of just one of the spin-1 pair partners. If the pair were strongly coupled, a full-field signal would be seen with a Rabi-nutation frequency of $2 \gamma \mathrm{B}_{1}$ and a beating frequency of $\sqrt{6} \gamma B_{1}{ }^{29}$ at high $B_{1}$ strengths due to the $\Delta \mathrm{m}= \pm 1$ transitions within the five states of a strongly coupled $S=2$ pair. We see only a $\gamma B_{1}$ Rabi-nutation frequency for all applied driving field strengths. This is not expected from this model, and thus we can exclude triplet-triplet annihilation and the trion model as an explanation of the observed electrically detected signal.

\section{Triplet-exciton polaron (TEP)}

The TEP mechanism results from the interaction between a triplet exciton and a free polaron. ${ }^{18,30}$ The process is similar to the trion; recombination leads to an excited free polaron with a higher mobility. However, unlike the trion process, the two pair partners are weakly spin coupled. The spin dependency of the triplet recombination results from the transition of the six-state manifold of the $s=1 / 2, s=1$ pair into a doublet [singlet $s=0, s=1 / 2$ ] system. The occurrence of a halffield resonance is predicted due to the $\Delta \mathrm{m}= \pm 2$ transitions within the triplet manifold, which become allowed because of strong dipolar interaction within the exciton. The resonance at half field affects the recombination rate and thus the current. Finally, a single spin-1/2 or $\gamma B_{1}$ Rabi-nutation frequency at full field due to the $\Delta \mathrm{m} \pm 1$ transitions of the free polaron is also expected. In contrast to the other mechanisms discussed previously, the presence of the correlated $s=1 / 2$ and $s=1$ nutation components in the presented data is consistent with the TEP model.
*Corresponding authors: boehme@physics.utah.edu, john.lupton@ physik.uni-regensburg.de

${ }^{1}$ M. Pope and C. E. Swenberg, Electronic Processes in Organic Crystals and Polymers (Clarendon, Oxford, 1982).

${ }^{2}$ V. A. Dediu, L. E. Hueso, I. Bergenti, and C. Taliani, Nat. Mater. 8, 707 (2009).

${ }^{3}$ E. L. Frankevich, A. A. Lymarev, I. Sokolik, F. E. Karasz, S. Blumstengel, R. H. Baughman, and H. H. Hörhold, Phys. Rev. B 46, 9320 (1992).

${ }^{4}$ J. Kalinowski, J. Szmytkowski, and W. Stampor, Chem. Phys. Lett. 378, 380 (2003).
${ }^{5}$ P. A. Bobbert, T. D. Nguyen, F. W. A. van Oost, B. Koopmans, and M. Wohlgenannt, Phys. Rev. Lett. 99, 216801 (2007).

${ }^{6}$ J. M. Lupton and C. Boehme, Nat. Mater. 7, 598 (2008).

${ }^{7}$ M. Reufer, M. J. Walter, P. G. Lagoudakis, A. B. Hummel, J. S. Kolb, H. G. Roskos, U. Scherf, and J. M. Lupton, Nat. Mater. 4, 340 (2005).

${ }^{8}$ G. Salis, S. F. Alvarado, M. Tschudy, T. Brunschwiler, and R. Allenspach, Phys. Rev. B 70, 085203 (2004).

${ }^{9}$ M. P. Delmo, S. Yamamoto, S. Kasai, T. Ono, and K. Kobayashi, Nature 457, 1112 (2009).

${ }^{10}$ U. E. Steiner and T. Ulrich, Chem. Rev. 89, 51 (1989). 
${ }^{11}$ M. Wohlgenannt, K. Tandon, S. Mazumdar, S. Ramasesha, and Z. V. Vardeny, Nature 409, 494 (2001).

${ }^{12}$ M. Wohlgenannt, W. Graupner, G. Leising, and Z. V. Vardeny, Phys. Rev. Lett. 82, 3344 (1999).

${ }^{13}$ L. S. Swanson, J. Shinar, A. R. Brown, D. D. C. Bradley, R. H. Friend, P. L. Burn, A. Kraft, and A. B. Holmes, Phys. Rev. B 46, 15072 (1992).

${ }^{14}$ M.-K. Lee, M. Segal, Z. G. Soos, J. Shinar, and M. A. Baldo, Phys. Rev. Lett. 94, 137403 (2005).

${ }^{15}$ D. R. McCamey, H. A. Seipel, S.-Y. Paik, M. J. Walter, N. J. Borys, J. M. Lupton, and C. Boehme, Nat. Mater. 7, 723 (2008).

${ }^{16}$ D. R. McCamey, K. J. van Schooten, W. J. Baker, S.-Y. Lee, S.-Y. Paik, J. M. Lupton, and C. Boehme, Phys. Rev. Lett. 104, 017601 (2010).

${ }^{17}$ D. R. McCamey, S.-Y. Lee, S.-Y. Paik, J. M. Lupton, and C. Boehme, Phys. Rev. B 82, 125206 (2010).

${ }^{18}$ V. Ern and R. E. Merrifield, Phys. Rev. Lett. 21, 609 (1968).

${ }^{19}$ E. J. W. List, C.-H. Kim, A. K. Naik, U. Scherf, G. Leising, W. Graupner, and J. Shinar, Phys. Rev. B 64, 155204 (2001).

${ }^{20}$ M. Gross, D. C. Muller, H.-G. Nothofer, U. Scherf, D. Neher, C. Brauchle, and K. Meerholz, Nature 405, 661 (2000).

${ }^{21}$ J. Scott, S. Carter, S. Karg, and M. Angelopoulos, Synth. Met. 85, 1197 (1997).
${ }^{22}$ Y. Cao, G. Yu, C. Zhang, R. Menon, and A. J. Heeger, Synth. Met. 87, 171 (1997).

${ }^{23}$ J. C. Scott, G. G. Malliaras, W. D. Chen, J.-C. Breach, J. R. Salem, P. J. Brock, S. B. Sachs, and C. E. D. Chidsey, Appl. Phys. Lett. 74, 1510 (1999).

${ }^{24}$ N. M. Atherton, Principles of Electron Spin Resonance (Ellis Horwood PTR Prentice Hall, Chichester, England, 1993).

${ }^{25}$ J. Behrends, A. Schnegg, K. Lips, E. A. Thomsen, A. K. Pandey, I. D. W. Samuel, and D. J. Keeble, Phys. Rev. Lett. 105, 176601 (2010).

${ }^{26}$ S.-Y. Lee, S.-Y. Paik, D. R. McCamey, J. Yu, P. L. Burn, J. M. Lupton, and C. Boehme, J. Am. Chem. Soc. 133, 2019 (2011).

${ }^{27}$ C. Boehme, Dynamics of Spin-Dependent Charge Carrier Recombination. Ph.D. thesis (Philipps-Universität Marburg, Germany 2002).

${ }^{28}$ V. M. Agranovich, D. M. Basko, K. Schmidt, G. C. LaRocca, F. Bassani, S. Forrest, K. Leo, and D. Lidzey, Chem. Phys. 272, 159 (2001).

${ }^{29}$ A. V. Astashkin and A. Schweiger, Chem. Phys. Lett. 174, 595 (1990).

${ }^{30}$ Y. Meng, X. J. Liu, B. Di, and Z. An, J. Chem. Phys. 131, 244502 (2009). 